\title{
Stable Suspension and Dispersion-Induced Transitions from Repulsive Casimir Forces Between Fluid-Separated Eccentric Cylinders
}

\section{Citation}

Rodriguez, Alejandro W., J. N. Munday, J. D. Joannopoulos, Federico Capasso, Diego A. R. Dalvit, and Steven G. Johnson. 2008. "Stable Suspension and Dispersion-Induced Transitions from Repulsive Casimir Forces Between Fluid-Separated Eccentric Cylinders." Physical Review Letters 101 (19). https://doi.org/10.1103/physrevlett.101.190404.

\section{Permanent link}

http://nrs.harvard.edu/urn-3:HUL.InstRepos:41371468

\section{Terms of Use}

This article was downloaded from Harvard University's DASH repository, and is made available under the terms and conditions applicable to Other Posted Material, as set forth at http:// nrs.harvard.edu/urn-3:HUL.InstRepos:dash.current.terms-of-use\#LAA

\section{Share Your Story}

The Harvard community has made this article openly available.

Please share how this access benefits you. Submit a story.

\section{Accessibility}




\title{
Stable suspension and dispersion-induced transitions from repulsive Casimir forces between fluid-separated cylinders
}

\author{
Alejandro W. Rodriguez, ${ }_{1}$ J. N. Munday, ${ }^{2}$ J. D. Joannopoulos, ${ }^{1}$ Federico Capasso, ${ }^{3}$ Diego A. R. Dalvit, ${ }^{4}$ and Steven G. Johnson ${ }^{5}$ \\ ${ }^{I}$ Department of Physics, Massachusetts Institute of Technology, Cambridge, MA 02139 \\ ${ }^{2}$ Department of Physics, Harvard University, Cambridge, MA 02139 \\ ${ }^{3}$ School of Engineering and Applied Sciences, Harvard University, Cambridge, MA 02139 \\ ${ }^{4}$ Theoretical Division, Los Alamos National Laboratory, Los Alamos, NM 87545 \\ ${ }^{5}$ Department of Mathematics, Massachusetts Institute of Technology, Cambridge, MA 02139
}

\begin{abstract}
We numerically demonstrate a stable mechanical suspension of a silica cylinder within a metallic cylinder separated by ethanol, via a repulsive Casimir force between the silica and the metal. We investigate cylinders with both circular and square cross sections, and show that the latter exhibit a stable orientation as well as a stable position, via a method to compute Casimir torques for finite objects. Furthermore, the stable orientation of the square cylinder undergoes a $45^{\circ}$ transition as the separation length-scale is varied, which is explained as a consequence of material dispersion.
\end{abstract}

The Casimir force, arising from quantum fluctuations of the electromagnetic field, was first described as an attractive, monotonically decaying force between metallic plates [1], but repulsive interactions can arise in special circumstances, e.g. involving fluid-separated asymmetric plates [2]. It has been proposed that repulsive Casimir forces between fluidseparated objects can lead to stable mechanical equilibria, and hence frictionless static bearings or other interesting passivesuspension devices [3, 4]. However, previous calculations and experiments involving fluid-separated objects have been restricted to geometries involving parallel plates or approximations thereof [5] (similar to work on air-separated metals, as reviewed in several recent papers, e.g. Ref. 6). Here, using recently developed numerical techniques [7], we present theoretical calculations of Casimir forces/torques between fluidseparated objects with finite square and circular cross sections (Fig. 1 insets) that rigorously demonstrate stable positional/orientational Casimir equilibria. (Vacuum-separated perfect-metal circular cylinders were shown to exhibit an unstable positional equilibrium [8].) In the case of square cross sections, a surprising result is obtained for the rotational equilibria: the stable orientation changes by $45^{\circ}$ depending on the lengthscale, which we show to be a consequence of material dispersion. In particular, for certain fluid-separated materials, the frequency dependence of the permittivity $\varepsilon$ (material dispersion) causes the Casimir force to switch from repulsive to attractive at some critical separation [2]; this leads to the orientation transition described here, and may produce other lengthscale-based qualitative transitions in future geometries. We present a method to compute Casimir torques on finite objects (in contrast to previous uncontrolled approximations [9] or analytical results for planar cases [3, 10, 11, 12, 13, 14]); we supplement the accurate results with a heuristic model based on the proximity force approximation (PFA) that turns out to capture qualitative behaviors of the orientation transition, and therefore provides some simple insight.

A repulsive Casimir force arises between parallel plates of $\varepsilon_{1,2}$ separated by a fluid of $\varepsilon_{f}$, if $\varepsilon_{1}(i \xi)<\varepsilon_{f}(i \xi)<\varepsilon_{2}(i \xi)$ for a sufficiently wide range of imaginary frequencies $\xi=$

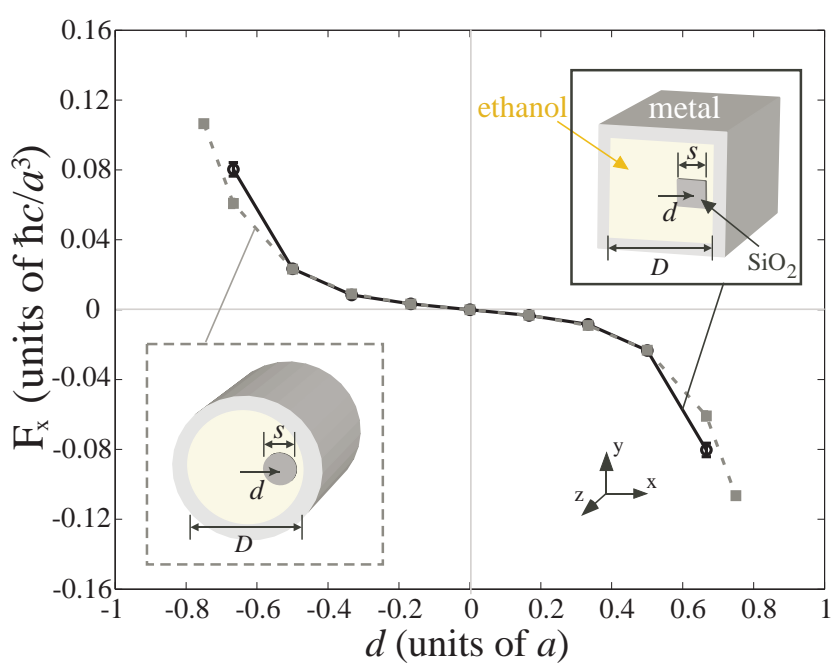

FIG. 1: Casimir force $F_{x}$ in the $x$-direction, per unit $z$-length, on a $\mathrm{SiO}_{2}$ cylinder suspended within a perfectly-metallic cylinder (inset), separated by fluid (ethanol), as a function of the $x$-displacement from equilibrium (eccentricity) $d$ [in units of $a=0.5(D-s)$ ] for both circular (solid-line) and square (dashed-line) cylinders. $d=0$ is seen to be a stable equilibrium.

$\operatorname{Im} \omega$ [2]. Three such materials are silica $\left(\mathrm{SiO}_{2}\right)$ and metal (Au) separated by ethanol [4], as discussed below. We studied the three-dimensional constant cross-section geometries shown in the inset of Fig. 1. square or circular $\mathrm{SiO}_{2}$ cylinders of diameter $s$ surrounded by a metal cylinder of diameter $D$, separated by ethanol. For computational ease, we use a perfect metal $[\varepsilon(i \xi) \rightarrow \infty$ ] for the outer cylinder, but we use experimental $\omega$-dependent $\varepsilon$ for the $\mathrm{SiO}_{2}$ and ethanol. In such cases, with object sizes and curvatures comparable to their separation, approximations of the Casimir force as a pairwise attraction between surfaces are not valid and their qualitative predictions (e.g. stability) may be incorrect [7, 15, 16].

Figure 1 shows the force per unit $z$-length on the inner cylinder as a function of the displacement $d$ from equilibrium in units of $a$, where $a \equiv 0.5(D-s)=0.0955 \mu \mathrm{m}$ so that $d / a= \pm 1$ for touching surfaces, for parameters $s / D=0.25$. 


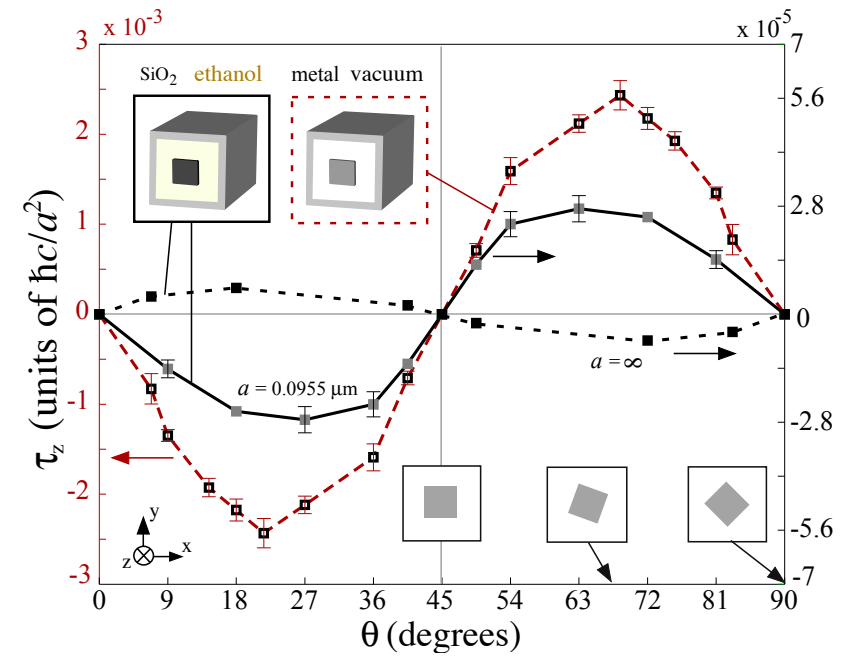

FIG. 2: Casimir torque $\tau_{z}$, per unit $z$-length, on the inner square rod of eccentric square cylinders as a function of the angle $\theta$ with respect to the $x$-axis (see right insets) for two material choices as shown in the top insets. Error bars are estimates of the effect of finite grid resolution.

It demonstrates a stable equilibrium for both square (solid black) and circular (dashed grey) cross sections. The computational method is based on integration of the mean electromagnetic stress tensor (valid even for fluids [17]) evaluated in terms of the imaginary-frequency Green's function via the fluctuation-dissipation theorem [7]. Additional details are provided below.

Having demonstrated positional stability (Fig. 1), we now explore an unusual effect arising from material dispersion. The square-cylinder geometry (Fig. 2 inset) must, by symmetry, exhibit two equilibrium orientations $\left(\theta=0^{\circ}\right.$ and $\theta=45^{\circ}$ ). A priori, it is not clear which of the two configurations is stable. To determine this, Fig. 2 plots the Casimir torque per unit $z$-length on the inner square, as a function of the rotation angle $\theta$ between the inner and outer squares. In addition to computing the torque for the $\mathrm{SiO}_{2}$-ethanolperfect-metal case, we also analyze the torque for vacuumseparated perfect-metal cylinders, in which case the forces are purely attractive and there is no stable positional equilibrium [8].

The resulting torque per unit $z$-length is shown in Fig. 2, for both the repulsive (solid, filled-square-lines) and attractive (solid, open-square-line) cases, as $\theta$ is varied from $-45^{\circ}$ to $45^{\circ}$. The stable orientation for both cases is $\theta=45^{\circ}$, a surprising result considering the difference in the sign of the force. A stable orientation of $45^{\circ}$ in the perfect-metal case is not so surprising, since an attractive force that decreases with separation should intuitively (in the heuristic picture of pairwise attractions between surfaces) favor an orientation where the surfaces are as close as possible, pulling the corners of the inner square towards the outer surfaces. On the other hand, a repulsive force should intuitively push the surfaces as far apart as possible, which would suggest a $0^{\circ}$ stable orienta-

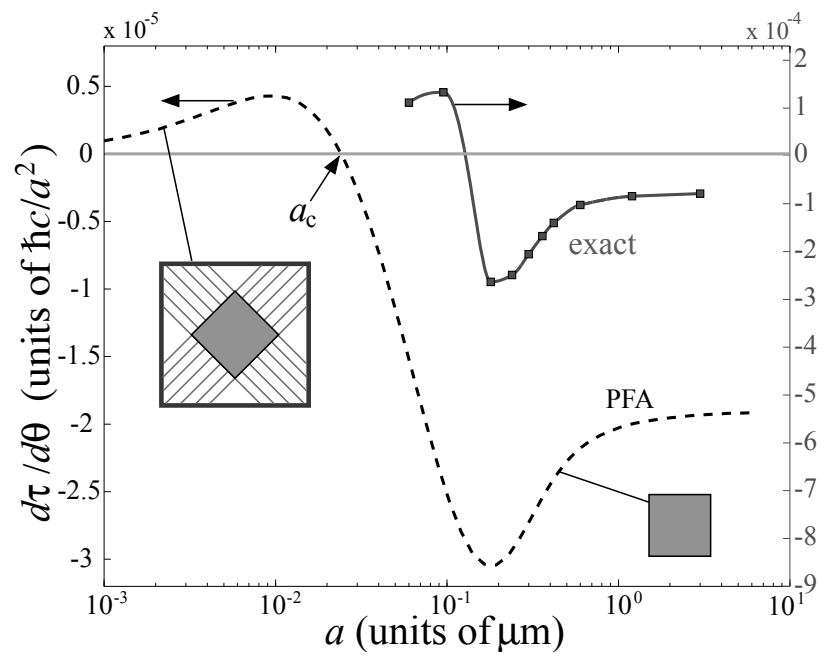

FIG. 3: Derivative of the Casimir torque in the $z$-direction with respect to $\theta, d \tau_{z} / d \theta$, in units of $\hbar c / a^{2}$, evaluated at $\theta \approx 0^{\circ}$, as a function of lengthscale $a=0.5(D-s)$. The solid line is a fit to the exact Casimir torque, computed by our numerical method (solid squares), and the dashed line is the torque as computed by a PFA approximation. Both curves display a change (dispersion-induced transition) in the stable orientation of the square.

tion. The reason for this apparent contradiction between intuition and the numerical results lies in the effects of material dispersion—as explained below, $\varepsilon_{\mathrm{Si}}-\varepsilon_{\text {eth }}$ (and hence the force) switches sign at some lengthscales (some $1 / \xi$ ). For example, if we neglect the material dispersion of $\mathrm{SiO}_{2}$ and ethanol, and use only the $\xi \rightarrow 0$ dielectric constants, the torque as a function of $\theta$ (shown as the dashed black line in Fig. 2) indeed exhibits a stable orientation at $0^{\circ}$, as expected from the pairwise intuition: in this case, the force is repulsive at all lengthscales.

Since $\theta=0^{\circ}$ is unstable for the $a=0.0955 \mu \mathrm{m}$ fluid case and stable for $a \rightarrow \infty$ (equivalent to $\xi \rightarrow 0$ ), there must be a transition at some critical intermediate lengthscale $a_{c}$. One way to determine $a_{c}$ is to calculate the derivative of the torque at $\theta=0^{\circ}$ as a function of $a$ (noting that the dimensionless torque $\tau a^{2} / \hbar c$ and its derivative go to a nonzero value as $a \rightarrow \infty$ ), and to look for a change in the sign. This derivative is plotted as the solid line in Fig. 3 and displays the expected transition from unstable $\left(d \tau_{z} / d \theta>0\right.$, left) to stable $\left(d \tau_{z} / d \theta<0\right.$, right $)$ at $a_{c} \approx 0.1 \mu \mathrm{m}$, a consequence of material dispersion. A better understanding of this transition can be gained by inspecting a simple heuristic (PFA). PFA is only an ad hoc model, in which the force on each point of the surface (and hence the torque) is treated as simply the parallel-plate (Lifshitz) force between fluid-separated half-spaces (with "lines of interaction" perpendicular to the inner square, as depicted in the left inset of Fig. 3). This simple model turns out to capture some qualitative features of the orientation transition, as shown by the dashed line in Fig. 3 , although it is of course quantitatively incorrect [11].

PFA provides an explanation for why orientational stability 
need not coincide with positional stability. Stability, in general, arises from the competing interactions of the inner and outer surfaces, e.g. in the $45^{\circ}$ orientation for repulsive interactions, the nearest surface "pushes" the corner away, while the other surface "pushes" the corner back. Which of these competing forces dominates depends on their sign and power law, but in the torque $\boldsymbol{\tau}=\mathbf{r} \times \mathbf{F}$ case, there is an additional effect (from the $\mathbf{r} \times$ dependence): the force "from" the nearest surface elements is parallel to the radial direction and does not produce a torque, whereas it does contribute to the total force. Therefore, the net force and torque can respond differently to the same material dispersion, because the lengthscales of the dominant contributing separations differ. More generally, in the exact model where the force cannot be decomposed into additive contributions, the dominant imaginary-frequency contribution can differ between torque and force.

There is another interesting feature in Fig. 3. for $a>a_{c}$, the derivative of the torque is nonmonotonic, decreasing in magnitude towards $a \rightarrow \infty$. This may seem counter-intuitive because the $\varepsilon$ contrast is maximum for $\xi \rightarrow 0$ (Fig. 4 inset), which would seem to predict greater forces. However, because the net torque arises from a competition between nearby and faraway surfaces, the magnitude depends not only on the force but also on the power law: if the force decreases more rapidly with distance, then faraway surfaces contribute less and the net torque is larger. This is precisely what is happening here: at intermediate $a$, material dispersion acts to increase the power law [2] compared to the $a \rightarrow \infty$ case where dispersion plays no role. Preliminary work shows similar effects in the positional stability. This also explains the relative torque magnitudes for $a \rightarrow \infty$ and $a=0.0955 \mu \mathrm{m}$ in Fig. 2 .

Since the qualitative behavior of the orientation transition is captured by PFA, it makes sense to examine a simple onedimensional model in detail, depicted on the inset of Fig. 4. a $\mathrm{SiO}_{2}$ slab of thickness $s$, separated from two metallic halfspaces, a (surface-to-surface) distance $a \equiv 0.5(D-s)$ from the $\mathrm{SiO}_{2}$ surface, with ethanol (fluid) in between. This geometry is analyzed using a generalization of the Lifshitz formula [18]. Figure 4 shows the force per unit area on the inner slab (again using $s / D=0.25$ ), as a function of the displacement $d$, for two lengthscales: $a=0.0955 \mu \mathrm{m}$ (red), and $a=0.6 \mu \mathrm{m}$ (black). There is a stable equilibrium at $d=0$, just as for the two-dimensional case in Fig. 1. In the case of perfect-metal half-spaces, the force changes sign at a critical distance $d_{c}$ that depends on the lengthscale $a$. However, if we use a realistic metal $(\mathrm{Au})$, shown by the dashed line for $a=0.0955 \mu \mathrm{m}$, this transition disappears and the force is always repulsive. These features are explained by the inset of Fig. 4, which shows $\varepsilon(i \xi)$ for $\mathrm{SiO}_{2}$, ethanol, and $\mathrm{Au}$. The key point is that the contributions to the Casimir force come primarily from imaginary "wavelengths" $2 \pi / \xi$ larger than some lengthscale set by the separation, while very short wavelengths (large $\xi$ ) on this scale are exponentially cut off in the force integral [19]. Thus, for large lengthscales $a$, the force is dominated by small- $\xi$ contributions where $\varepsilon_{\text {silica }}<$ $\varepsilon_{\text {ethanol }}<\varepsilon_{\text {metal }}$, giving a repulsive force and a stable equi-

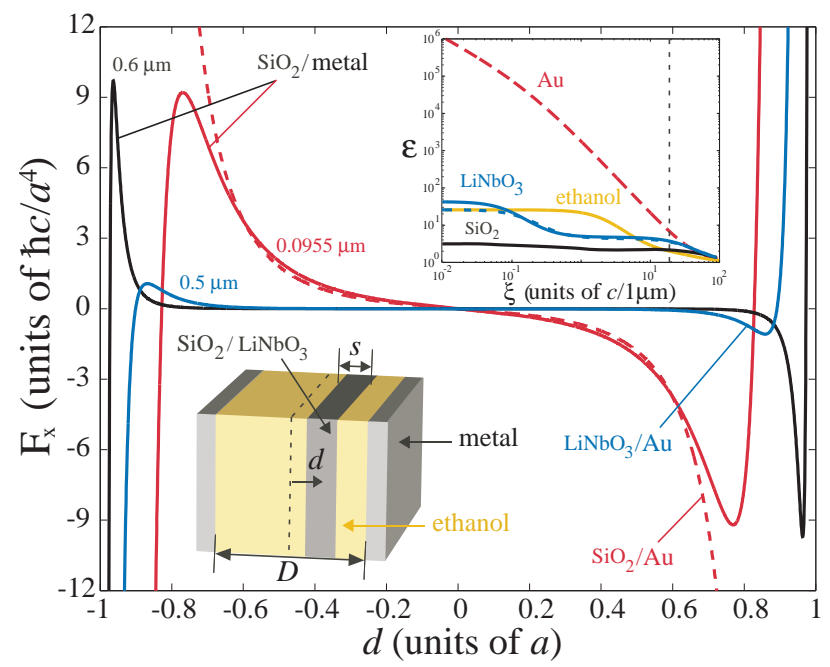

FIG. 4: Casimir force $F_{x}$ in the $x$-direction, per unit area, between a planar $\mathrm{SiO}_{2}$ slab suspended between two perfect-metal plates (solid red and black lines) or gold half-spaces (dashed red line), separated by a fluid (ethanol), as a function of the dimensionless $x$-displacement $d$ from equilibrium. The force is plotted also at two lengthscales $a \equiv 0.5(D-s)=0.0955 \mu \mathrm{m}$ (red lines) and $a=0.6 \mu \mathrm{m}$ (black line). The solid blue line shows the same quantity for the case of a lithium-niobate slab and a gold half-space, for $a=0.5 \mu \mathrm{m}$. (Insets:) Top inset: $\varepsilon(i \xi)$ for $\mathrm{SiO}_{2}$ (black), $\mathrm{LiNbO}_{3}$ (solid and dashed blue), ethanol (orange) and gold (red) as a function of imaginary frequency $\xi$. Bottom inset: schematic of geometry.

librium. On the other hand, for small lengthscales and separations, large- $\xi$ contributions become more important, for which $\varepsilon_{\text {silica }}>\varepsilon_{\text {ethanol }}$ (for $\xi>2.3 \mathrm{c} / \mu \mathrm{m}$, marked by the vertical dashed line in the inset of Fig. 4, leading to attractive forces. For the case of perfect-metal cylinders, these attractive contributions are large enough to flip the sign of the total force for small separations, whereas for Au the attractive high- $\xi$ contributions are suppressed by the diminishing $\varepsilon(i \xi)$ of the $\mathrm{Au}$ and the force remains repulsive. While the transition is absent in the $\mathrm{SiO}_{2}$-ethanol-Au configuration, we have calculated the force between ethanol-separated lithium-niobate $\mathrm{LiNbO}_{3}$ and Au plates and found a sign transition at separations $\sim 0.05 \mu \mathrm{m}$. This is illustrated in Fig. 4 (solid blue line); because $\mathrm{LiNbO}_{3}$ 's anisotropy greatly complicates the modeling, we used a PFA approximation based on summing the Lifshitz forces between semi-infinite $\mathrm{Au}$ and $\mathrm{LiNbO}_{3}$ slabs (neglecting the finite $\mathrm{LiNbO}_{3}$ thickness). The two principal values of the $\varepsilon$ tensor of $\mathrm{LiNbO}_{3}$ [20] are plotted in the inset. There are also transitions for ethanol-separated bariumtitanate and calcite plates at separations $\sim 0.01 \mu \mathrm{m}$ [3]. Since these two cases are much more difficult to compute (the outer cylinder is not perfect metal and $\mathrm{LiNbO}_{3}$ is anisotropic), we focused on the $\mathrm{SiO}_{2}$-ethanol-metal case here, which has similar qualitative behaviors.

The experimental $\varepsilon \mathrm{s}$ of $\mathrm{SiO}_{2}$ and ethanol were fit to a standard multiple-oscillator model [21] accurate from infrared to ultraviolet wavelengths: $\varepsilon(i \xi)=1+$ 
$\sum_{n=1}^{N} C_{n}\left[1+\left(\xi / \omega_{n}\right)^{2}\right]^{-1}$. The parameters we used are [20, 22]: ethanol $(N=2) \omega_{n}=\{6.6,114\} \times 10^{14} \mathrm{~Hz}$ and $C_{n}=$ $\{23.84,0.852\} ; \mathrm{SiO}_{2}(N=3) \omega_{n}=\{0.867,1.508,203.4\} \times$ $10^{14} \mathrm{~Hz}$ and $C_{n}=\{0.829,0.095,1.098\}$. We model the dielectric constant of Au by a Drude model $\varepsilon(i \xi)=1+$ $\omega_{p}^{2} / \xi(\xi+\gamma)$, where $\omega_{p}=1.367 \times 10^{14} \mathrm{~Hz}$ and $\gamma=$ $5.320 \times 10^{13} \mathrm{~Hz}$ [20]. The Casimir force $\mathbf{F}$ is computed as an integral $\mathbf{F} \sim \int_{0}^{\infty} d \xi \oiiint\langle\mathbf{T}\rangle d \mathbf{A}$ [7], where the mean stress tensor $\langle\mathbf{T}\rangle$ is computed at each position and frequency from the Green's function as described in Ref. 7. The Casimir torque $\tau$, was computed in the same framework, with a minor modification, $\boldsymbol{\tau} \sim \int_{0}^{\infty} d \xi \oiiint \mathbf{r} \times(\langle\mathbf{T}\rangle d \mathbf{A})$, also proposed in our earlier work [7].

We are hopeful that these phenomena will be amenable to experiment. There are some experimental advantages over planar geometries: the concentric configuration is a stable equilibrium, static charges on the outer metallic cylinder are screened in the interior, and the equilibrium is easily distinguished from electrostatic effects (which cannot produce stability [15]). (If we inverted the geometry, to have the $\mathrm{SiO}_{2}$ on the outside and the metal on the inside, then the larger static $\varepsilon$ of the fluid compared to the $\mathrm{SiO}_{2}$ would lead to a classical stable equilibrium for a charged inner cylinder [15, 23, 24].) We expect that similar stability will be obtained for real metals, as in our Au calculation in Fig. 4, and there are several materials that exhibit repulsive-attractive transitions (unlike $\mathrm{SiO}_{2}-$ ethanol-Au) that should display the orientation transition. We have also uncovered an intriguing question to explore in future work: in the PFA heuristic, it appears that the critical lengthscale at which the orientation transition occurs is smaller than the corresponding positional transition lengthscale (changing from stable to unstable suspension), whereas the exact calculations give the opposite result.

This work was supported in part by U. S. Dept. of Energy Grant \#DE-FG02-97ER25308, the NSF MRSEC program under Grant \#DMR-0213282, and by the MIT Ferry Fund.

[1] H. B. G. Casimir, Proc. K. Ned. Akad. Wet. 51, 793 (1948).
[2] I. E. Dzyaloshinskiı̌, E. M. Lifshitz, and L. P. Pitaevskiı̌, Adv. Phys. 10, 165 (1961).

[3] J. N. Munday, D. Iannuzzi, Y. Barash, and F. Capasso, Phys. Rev. A 71, 042102 (2005).

[4] F. Capasso et al., IEEE J. Selected Topics in Quant. Elec. 13, 400 (2007).

[5] J. N. Munday and F. Capasso, Phys. Rev. A 75, 060102(R) (2007).

[6] M. Bordag et al. Phys. Rep. 353, 1 (2001). K. A. Milton, Journal of Physics A: Mathematical and General 37, R209 (2004). S. K. Lamoreaux, Rep. Prog. Phys. 68 (2005).

[7] A. Rodriguez et al., Phys. Rev. A 76, 032106 (2007).

[8] D. A. R. Dalvit et al., Phys. Rev. A 74, 020101(R) (2006).

[9] A. Scardicchio and R. L. Jaffe, Nuclear Physics B. 704, 552 (2005).

[10] S. J. van Enk, Phys. Rev. A 52, 2569 (1995).

[11] R. B. Rodrigues et al., Europhys. Lett. 75, 822 (2006).

[12] V. A. Parsegian and G. H. Weiss, J. Adhes. Sci. Technol. 3, 259 (1972).

[13] C.-G. Shao, A.-H. Tong, and J. Luo, Phys. Rev. A 72, 022102 (2005).

[14] H. Razmi and S. M. Modarresi, Int. J. Theor. Phys. 44, 229 (2005).

[15] D. A. R. Dalvit et al., Europhys. Lett. 67, 517 (2004).

[16] H. Gies and K. Klingmuller, Phys. Rev. Lett. 96, 220401 (2006).

[17] L. P. Pitaevskiı̌, Phys. Rev. A 73, 047801 (2006).

[18] M. S. Tomaš, Phys. Rev. A 66, 052103 (2002).

[19] E. M. Lifshitz, Sov. Phys. JETP 2, 73 (1956).

[20] L. Bergstrom, Adv. Colloid and Interface Science 70, 125 (1997).

[21] J. Mahanty and B. W. Ninham, Dispersion forces (Academic London, 1976).

[22] A. Milling, P. Mulvaney, and I. Larson, J. Colloid and Interface Science 180, 460 (1996).

[23] R. Y. Chiao and J. Boyce, Phys. Rev. Lett. 73, 3383 (1994).

[24] P. W. Milonni, D. F. V. James, and H. Fearn, Phys. Rev. Lett. 75, 3194 (1995). 